\title{
Geometric Invariant Theory based on Weil divisors
}

\author{
Jürgen Hausen
}

\begin{abstract}
Given an action of a reductive group on a normal variety, we describe all invariant open subsets admitting a good quotient with a quasiprojective or a divisorial quotient space. We obtain several new Hilbert-Mumford type theorems, and we extend a projectivity criterion of Białynicki-Birula and Święcicka for varieties with semisimple group action from the smooth to the singular case.
\end{abstract}

\section{Introduction}

This paper is devoted to a central task of geometric invariant theory (GIT), formulated in [BB02]: Given an action of a reductive group $G$ on a normal variety $X$, describe all $G$-invariant open subsets $U \subset X$ admitting a good quotient, which means a $G$-invariant affine morphism $U \rightarrow U / / G$ such that the structure sheaf of $U / / G$ equals the sheaf of invariants $p_{*}\left(\mathcal{O}_{U}\right)^{G}$. We call these $U$ for the moment the $G$-sets.

In [MFK94], Mumford obtains $G$-sets with quasiprojective quotient spaces. Given a $G$-linearized line bundle $L \rightarrow X$, which means that $G$ acts on the total space making the projection equivariant and inducing linear maps on the fibres, he calls a point $x \in X$ semistable if some positive power of $L$ admits a $G$-invariant section $f$ such that removing the zeroes gives an affine neighbourhood $X_{f}$ of $x$.

The set $X^{\mathrm{ss}}(L)$ of semistable points of a $G$-linearized line bundle $L$ admits a good quotient $X^{\mathrm{ss}}(L) \rightarrow X^{\mathrm{ss}}(L) / / G$ with a quasiprojective quotient space. For smooth $X$, basically all quasiprojective quotient spaces arise in this way: every $G$-set $U$ with $U / / G$ quasiprojective is $G$-saturated in some $X^{\mathrm{ss}}(L)$, which means that $U$ is saturated with respect to the quotient map.

For singular $X$, Mumford's method does not provide all quasiprojective quotients (see Proposition 3.6). Here, replacing the bundles $L$ with Weil divisors $D$ yields a more rounded picture: We define a $G$-linearization of $D$ to be a certain lifting of the $G$-action to $\operatorname{Spec}(\mathcal{A})$, where $\mathcal{A}=$ $\bigoplus_{E \in \Lambda} \mathcal{O}(E)$ with $\Lambda=\mathbb{N} D$, and the set $X^{\mathrm{ss}}(D)$ of semistable points is the union of all affine sets $X_{f}$, where $f \in \mathcal{A}(X)$ is $G$-invariant and homogeneous of positive degree. The first result is Theorem 3.3, as follows.

Theorem. Let a reductive group $G$ act on a normal variety $X$.

i) For any $G$-linearized Weil divisor $D$ on $X$, there is a good quotient $X^{\mathrm{ss}}(D) \rightarrow X^{\mathrm{ss}}(D) / / G$ with a quasiprojective variety $X^{\mathrm{ss}}(D) / / G$.

ii) If $U \subset X$ is open, $G$-invariant, and has a good quotient $U \rightarrow U / / G$ with $U / / G$ quasiprojective, then $U$ is a $G$-saturated subset of some $X^{\mathrm{ss}}(D)$.

Received 20 May 2003, accepted in final form 28 November 2003, published online 15 October 2004.

2000 Mathematics Subject Classification 14L24, 14L30.

Keywords: reductive group action, linearization, good quotient, Hilbert-Mumford theorems.

This journal is (C) Foundation Compositio Mathematica 2004. 


\section{GIT BASED ON WEIL DIVISORS}

However, the quasiprojective quotient spaces are not the whole story by far, and a further aim is to complement also the picture developed in [Hau01] for divisorial quotient spaces, which means (possibly nonseparated) prevarieties $Y$ such that every $y \in Y$ has an affine neighbourhood $Y \backslash \operatorname{Supp}(E)$ with an effective Cartier divisor $E$ (see [Bor63] and [BGI71]). For the occurrence of nonseparatedness in quotient constructions, compare also [MFK94, Proposition 1.9], [Sum74, Corollary 1.3], and [ACH01].

To obtain divisorial quotient spaces, we work with finitely generated groups $\Lambda$ of Weil divisors. Similarly as before, $G$-linearization of such a $\Lambda$ is a lifting of the $G$-action to $\operatorname{Spec}(\mathcal{A})$, where now $\mathcal{A}=$ $\bigoplus_{D \in \Lambda} \mathcal{O}(D)$. We also have a notion of semistability, and the resulting statements generalize [Hau01] (see Theorem 3.5).

Theorem. Let a reductive group $G$ act on a normal variety $X$.

i) For any $G$-linearized group $\Lambda$ of Weil divisors on $X$, there is a good quotient $X^{\mathrm{ss}}(\Lambda) \rightarrow$ $X^{\mathrm{ss}}(\Lambda) / / G$ with a divisorial prevariety $X^{\mathrm{ss}}(\Lambda) / / G$.

ii) If $U \subset X$ is open, $G$-invariant, and has a good quotient $U \rightarrow U / / G$ with $U / / G$ divisorial, then $U$ is a $G$-saturated subset of some $X^{\mathrm{ss}}(\Lambda)$.

A simple example shows that, in general, the respective sets of semistable points of a single linearized divisor $D$ and the group $\mathbb{Z} D$ differ (compare [Hau01, Example 3.5]). Let $G:=\mathbb{C}^{*}$ act linearly on $X:=\mathbb{C}^{2}$ via

$$
t \cdot(z, w):=\left(t z, t^{-1} w\right)
$$

Consider the invariant $\operatorname{divisor} D:=\operatorname{div}(z)$ on $X$. Then $D$ as well as the group $\Lambda:=\mathbb{Z} D$ are canonically $G$-linearized, via the induced action of $G$ on the function field. According to the respective Definitions 3.2 and 3.4 of semistability, one obtains

$$
X^{\mathrm{ss}}(D)=\mathbb{C}^{*} \times \mathbb{C}, \quad X^{\mathrm{ss}}(\Lambda)=\mathbb{C}^{2} \backslash\{(0,0)\} .
$$

Moreover, let us have a look at the quotient spaces. For the first set, the quotient space is the affine line, whereas in the second case a true (divisorial) pre-variety occurs: the affine line with a doubled point.

For practical purposes, it is often helpful to perform the construction of $G$-sets by means of subtori of $G$. Classically, this is done by the Hilbert-Mumford Lemma [MFK94, Theorem 2.1]: for a $G$-linearized ample bundle $L$ on a projective variety $X$, it gives a semistability criterion in terms of one-parameter subgroups; here, we deal with the following version, involving a maximal torus $T \subset G$ (compare [BB02] and [Sch03]):

$$
X^{\mathrm{ss}}(L, G)=\bigcap_{g \in G} g \cdot X^{\mathrm{ss}}(L, T)
$$

In this form, the statement allows a far reaching generalization; in particular, the hypotheses of projectivity and ampleness can be dropped (see Theorem 4.1).

Theorem. Let a reductive group $G$ act on a normal variety $X$, and let $T \subset G$ be a maximal torus.

i) Let $D$ be a $G$-linearized Weil divisor on $X$. Then we have

$$
X^{\mathrm{ss}}(D, G)=\bigcap_{g \in G} g \cdot X^{\mathrm{ss}}(D, T) .
$$

ii) Let $\Lambda \subset \operatorname{WDiv}(X)$ be a $G$-linearized subgroup. Then we have

$$
X^{\mathrm{ss}}(\Lambda, G)=\bigcap_{g \in G} g \cdot X^{\mathrm{ss}}(\Lambda, T) .
$$




\section{J. Hausen}

Finally, in $\S 5$, we focus on the case of a semisimple group $G$. We ask for maximal $G$-sets (compare [BB02]): A qp-maximal $G$-set is a $G$-set $U \subset X$ with $U / / G$ quasiprojective such that $U$ does not occur as a $G$-saturated proper subset in some $U^{\prime} \subset X$ with the same properties. Similarly, a $d$-maximal $G$-set is a subset having the analogous properties with respect to divisorial quotient spaces.

Reducing the construction of these sets to the construction of the qp- and the d-maximal $T$-sets for a maximal torus $T \subset G$ amounts to tackling Białynicki-Birula's Conjecture [BB02, $\S 12.1$ ]: Given a maximal $T$-set $U \subset X$ which is invariant under the normalizer $N \subset G$ of $T$, he asks if the following set is open and admits a good quotient by $G$ :

$$
W(U):=\bigcap_{g \in G} g \cdot U .
$$

Here are the known positive results concerning qp- and d-maximal $T$-sets $U \subset X$ : The case of $G=\mathrm{SL}_{2}$ acting on a smooth $X$ is settled in [BBS92, Theorem 9] and [Hau03, Theorem 2.2]. If $U / / T$ is projective and $X$ is smooth, then [BBS95, Corollary 1] gives a positive answer for a general connected semisimple group $G$. Moreover, the problem is solved in the case $U=X$ (see [BB02, Theorem 12.4] and [Hau01, Theorem 5.1]). We show the following in Theorem 5.2 and Corollary 5.5.

Theorem. Let $G$ be a connected semisimple group, and $T \subset G$ a maximal torus with normalizer $N \subset G$. Let $X$ be a normal $G$-variety, $U \subset X$ an $N$-invariant open subset, and $W(U)$ the intersection of all translates $g \cdot U$, where $g \in G$.

i) If $U \subset X$ is a qp-maximal $T$-set, then $W(U)$ is open and $T$-saturated in $U$, and there is a good quotient $W(U) \rightarrow W(U) / / G$ with $W(U) / / G$ quasiprojective.

ii) If $U$ admits a good quotient $U \rightarrow U / / T$ with $U / / T$ projective, then $W(U)$ is open and $T$-saturated in $U$, and there is a good quotient $W(U) \rightarrow W(U) / / G$ with $W(U) / / G$ projective.

iii) If $U \subset X$ is a d-maximal $N$-set, then $W(U)$ is open and $T$-saturated in $U$, and there is a good quotient $W(U) \rightarrow W(U) / / G$ with $W(U) / / G$ divisorial.

In the setting of part ii, we can prove much more. It turns out that $U$ and $W(U)$ are the sets of semistable points of an ordinary linearized ample line bundle, and - even more surprising - that $X$ is projective. This extends the main result of [BBS95] from the smooth to the normal case and thus gives an answer to the problem discussed in [BBS95, Remark p. 965]. More precisely, we prove the following in Theorem 5.4.

Theorem. Let $G$ be a connected semisimple group, $T \subset G$ a maximal torus with normalizer $N \subset G$, and $X$ be a normal $G$-variety. Suppose that $U \subset X$ is $N$-invariant, open and admits a good quotient $U \rightarrow U / / T$ with $U / / T$ projective. Then there is an ample $G$-linearized line bundle $L$ on $X$ with $U=X^{\mathrm{ss}}(L, T)$, we have $X=G \cdot U$, and $X$ is projective.

\section{Polyhedral semigroups and $G$-linearization}

In this section, we transfer Mumford's concepts of [MFK94, $\S 1.3]$ to the framework of Weil divisors. We introduce polyhedral semigroups of Weil divisors, and define the notion of a $G$-linearization for such a semigroup. Moreover, we give a geometric interpretation of this concept, and provide basic statements concerning existence and uniqueness of linearizations.

Throughout the whole paper, we work over an algebraically closed field $\mathbb{K}$ of characteristic zero. In this section, $X$ denotes an irreducible normal prevariety over $\mathbb{K}$, which means that $X$ is an 


\section{GIT BASED ON WEIL DIVISORS}

integral, normal, but possibly nonseparated scheme of finite type over $\mathbb{K}$ (compare also [Hum81, $\S$ I.2.2]). The word 'point' always refers to a closed point.

By $\operatorname{WDiv}(X)$ we denote the group of Weil divisors of $X$, and $\operatorname{CDiv}(X) \subset \operatorname{WDiv}(X)$ is the subgroup of Cartier divisors. For a finitely generated subsemigroup $\Lambda \subset \operatorname{WDiv}(X)$, let $\Gamma(\Lambda) \subset$ $\operatorname{WDiv}(X)$ denote the subgroup generated by $\Lambda$. We say that the semigroup $\Lambda$ is polyhedral if it is the intersection of $\Gamma(\Lambda)$ with a convex polyhedral cone in $\mathbb{Q} \otimes_{\mathbb{Z}} \Gamma(\Lambda)$.

Fix a polyhedral semigroup $\Lambda \subset \operatorname{WDiv}(X)$. Since we assumed $X$ to be normal, there is an associated $\mathcal{O}_{X}$-module $\mathcal{O}_{X}(D)$ of rational functions for any $D \in \Lambda$. In fact, multiplication in the function field $\mathbb{K}(X)$ even gives rise to a $\Lambda$-graded $\mathcal{O}_{X}$-algebra:

$$
\mathcal{A}:=\bigoplus_{D \in \Lambda} \mathcal{A}_{D}:=\bigoplus_{D \in \Lambda} \mathcal{O}_{X}(D)
$$

Now, let $G$ be a linear algebraic group, and let $G$ act on $X$. That means in particular that this action is given by a morphism $\alpha: G \times X \rightarrow X$, and, denoting by $\mu: G \times G \rightarrow G$ the multiplication map, we have the following commutative diagram.

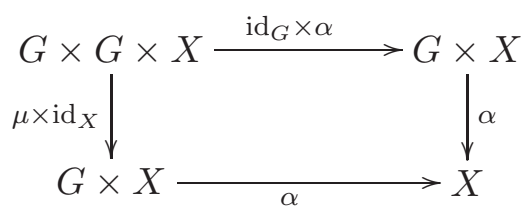

Similarly to [MFK94, Definition 1.6], the definition of a $G$-linearization of the semigroup $\Lambda \subset$ $\mathrm{WDiv}(X)$ is formulated in terms of $\mathcal{A}$, the above maps and the projection maps

$$
\begin{array}{cl}
\operatorname{pr}_{G \times X}: G \times G \times X \rightarrow G \times X, & \left(g_{1}, g_{2}, x\right) \mapsto\left(g_{2}, x\right), \\
\operatorname{pr}_{X}: G \times X \rightarrow X, & (g, x) \mapsto x .
\end{array}
$$

Definition 1.1. A $G$-linearization of $\Lambda$ is an isomorphism $\Phi: \alpha^{*} \mathcal{A} \rightarrow \operatorname{pr}_{X}^{*} \mathcal{A}$ of $\Lambda$-graded $\mathcal{O}_{G \times X^{-}}$ algebras such that $\Phi$ is the identity in degree zero, and the following diagram is commutative.

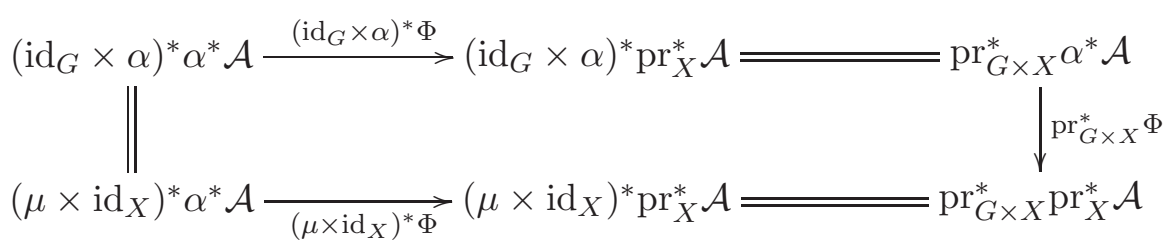

Note that if $\Lambda=\bigoplus_{n \geqslant 0} n D$ with a Cartier divisor $D$, then the $G$-linearizations $\Phi: \alpha^{*} \mathcal{A} \rightarrow \operatorname{pr}_{X}^{*} \mathcal{A}$ of $\Lambda$ correspond to the $G$-linearizations of the invertible sheaf $\mathcal{O}_{X}(D)$ in the sense of [MFK94, Definition 1.6] via passing to the corresponding map in degree one, $\Phi_{1}: \alpha^{*} \mathcal{O}_{X}(D) \rightarrow \operatorname{pr}_{X}^{*} \mathcal{O}_{X}(D)$.

In order to interpret Definition 1.1 geometrically, look at the scheme $\widetilde{X}:=\operatorname{Spec}(\mathcal{A})$ over $X$. Note that the $\Lambda$-grading of $\mathcal{A}$ defines an action of the torus $S:=\operatorname{Spec}(\underset{\widetilde{K}}{\mathbb{K}}[\Gamma(\Lambda)])$ on $\widetilde{X}$. We list some properties; for example, over the smooth locus, the canonical map $q: \widetilde{X} \rightarrow X$ is locally trivial with an affine toric variety as fibre.

Proposition 1.2. Let $U \subset X$ be an open subset such that every $D \in \Lambda$ is Cartier on $U$, and set $\widetilde{U}:=q^{-1}(U)$.

i) The map $q: \widetilde{U} \rightarrow U$ is locally trivial with typical fibre $\widetilde{U}_{x} \cong \operatorname{Spec}(\mathbb{K}[\Lambda])$. The open set $\widehat{U} \subset \widetilde{U}$ of free $S$-orbits is an $S$-principal bundle over $U$.

ii) The inclusion $\widehat{U} \subset \widetilde{U}$ corresponds to the inclusion $\mathcal{A} \subset \mathcal{B}$ of the graded $\mathcal{O}_{U}$-algebras $\mathcal{A}$ and $\mathcal{B}$ arising from $\Lambda$ and $\Gamma(\Lambda)$. 


\section{J. HAUSEN}

iii) For any homogeneous section $f \in \mathcal{A}(U)$, its zero set as a function on $\widehat{U}$ equals the set $\widehat{U} \cap$ $q^{-1}(\operatorname{Supp}(\operatorname{div}(f)+D))$.

Proof. Consider the group $\Gamma(\Lambda)$ generated by $\Lambda$ and its $\mathcal{O}_{X}$-algebra $\mathcal{B}$. Locally, $\mathcal{B}$ is a Laurent monomial algebra over $\mathcal{O}_{U}$, i.e. for small affine open $V \subset U$, we have a graded isomorphism over $\mathcal{O}(V)$ :

$$
\mathcal{B}(V) \cong \mathcal{O}(V) \otimes_{\mathbb{K}} \mathbb{K}[\Gamma(\Lambda)]
$$

Cutting this down to the subsemigroup $\Lambda \subset \Gamma(\Lambda)$ and the associated subalgebra $\mathcal{A} \subset \mathcal{B}$, we obtain local triviality of $q: \widetilde{U} \rightarrow U$. The remaining statements then follow easily.

Any $G$-linearization $\Phi: \alpha^{*} \mathcal{A} \rightarrow \operatorname{pr}_{X}^{*} \mathcal{A}$ of the polyhedral semigroup $\Lambda \subset \operatorname{WDiv}(X)$ defines the following commutative diagram.

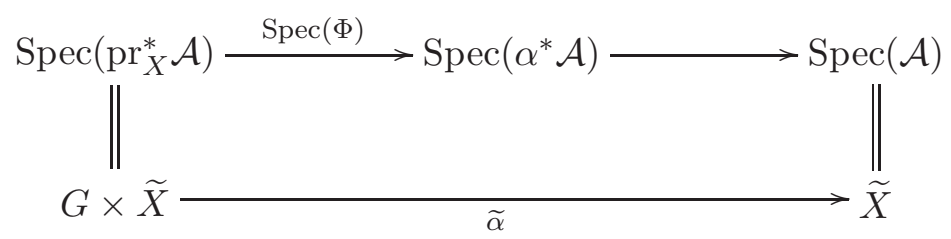

Note that $\operatorname{Spec}\left(\alpha^{*} \mathcal{A}\right)$ is the fibre product of $\alpha: G \underset{\widetilde{X}}{\times} X \rightarrow X$ and the canonical map $\tilde{X} \rightarrow X$. Then the upper right arrow is merely the projection to $\widetilde{X}$.

Proposition 1.3.

i) The map $\widetilde{\alpha}: G \times \widetilde{X} \rightarrow \widetilde{X}$ is a $G$-action that commutes with the $S$-action on $\widetilde{X}$, and makes the canonical map $\widetilde{X} \rightarrow X$ equivariant.

ii) For every action $\widetilde{\alpha}: G \times \widetilde{X} \rightarrow \widetilde{X}$ as in part $i$, there is a unique $G$-linearization $\Phi: \alpha^{*} \mathcal{A} \rightarrow \operatorname{pr}_{X}^{*} \mathcal{A}$ making the diagram (1) commutative.

Proof. For part i, note that $q \circ \widetilde{\alpha}$ equals $\alpha \circ\left(\operatorname{id}_{G} \times q\right)$, because $\Phi$ is the identity in degree zero. Moreover, the commutative diagram of Definition 1.1 yields the associativity law of a group action for $\widetilde{\alpha}$, and $e_{G} \in G$ acts trivially because $\Phi$ is an isomorphism. Finally, the actions of $G$ and $S$ commute, because $\widetilde{\alpha}$ has graded comorphisms.

To verify ii, we use that $\operatorname{Spec}\left(\alpha^{*} \underset{\widetilde{A}}{\mathcal{A}}\right)$ is the fibre product of $\alpha: G \times X \rightarrow X$ and $\widetilde{X} \rightarrow X$. By the universal property, $\widetilde{\alpha}: G \times \widetilde{X} \rightarrow \widetilde{X}$ lifts to a unique morphism $\operatorname{Spec}\left(\operatorname{pr}_{X}^{*} \mathcal{A}\right) \rightarrow \operatorname{Spec}\left(\alpha^{*} \mathcal{A}\right)$. It is straightforward to check that this morphism stems from a $G$-linearization $\Phi: \alpha^{*} \mathcal{A} \rightarrow \operatorname{pr}_{X}^{*} \mathcal{A}$.

Eventually, via the lifted $G$-action on $\widetilde{X}$, we associate to any $G$-linearization of $\Lambda$ a graded $G$-sheaf structure on $\mathcal{A}$. The latter is a collection of graded $\mathcal{O}(U)$-algebra homomorphisms $\mathcal{A}(U) \rightarrow \mathcal{A}(g \cdot U)$, $f \mapsto g \cdot f$, being compatible with group operations in $G$ and with restriction and algebra operations in $\mathcal{A}$; thereby $G$ acts as usual on the structure sheaf $\mathcal{O}_{X}$ via $g \cdot f(x):=f\left(g^{-1} \cdot x\right)$.

Proposition 1.4. Let $\Phi: \alpha^{*} \mathcal{A} \rightarrow \operatorname{pr}_{X}^{*} \mathcal{A}$ be a $G$-linearization. Then there is a unique graded $G$-sheaf structure on $\mathcal{A}$ satisfying $g \cdot f(\widetilde{x}):=f\left(g^{-1} \cdot \widetilde{x}\right)$ for any $\widetilde{x} \in \widetilde{X}$ lying over the smooth locus of $X$. For every $G$-invariant open $U \subset X$, the induced representation of $G$ on $\mathcal{A}(U)$ is rational.

Proof. Over the smooth locus of $X$, we may define the $G$-sheaf structure according to $g \cdot f(\widetilde{x}):=$ $f\left(g^{-1} \cdot \widetilde{x}\right)$. By normality, it uniquely extends to $X$. Rationality of the induced representations follows, for example, from [KKLV89, Lemma 2.5].

Remark 1.5. Let $\Phi: \alpha^{*} \mathcal{A} \rightarrow \operatorname{pr}_{X}^{*} \mathcal{A}$ be a $G$-linearization. Then a section $f \in \mathcal{A}(X)$ is invariant with respect to the induced $G$-representation on $\mathcal{A}(X)$ if and only if $\Phi\left(\alpha^{*}(f)\right)=\operatorname{pr}_{X}^{*}(f)$ holds. 


\section{GIT BASED ON WEIL DIVISORS}

We give two existence statements for $G$-linearizations. The first one is the analogue of Mumford's result [MFK94, Corollary 1.6] and [KKLV89, Proposition 2.4]. We use the following terminology: Given polyhedral semigroups $\Lambda^{\prime} \subset \Lambda$, we say that $\Lambda^{\prime}$ is of finite index in $\Lambda$ if there is a positive $n \in \mathbb{Z}$ with $n \Lambda \subset \Lambda^{\prime}$.

Proposition 1.6. Suppose that $X$ is separated and that $G$ is connected. Then, for any polyhedral semigroup $\Lambda \subset \mathrm{WDiv}(X)$, some subsemigroup $\Lambda^{\prime} \subset \Lambda$ of finite index admits a $G$-linearization.

Proof. By normality, it suffices to provide a $G$-linearization of $\mathcal{A}$ over the smooth locus. Hence, we may assume that $\Gamma \subset \operatorname{CDiv}(X)$ holds. Consider the group $\Gamma(\Lambda) \subset \operatorname{CDiv}(X)$ generated by $\Lambda$, and fix any basis $D_{1}, \ldots, D_{k}$ of $\Gamma(\Lambda)$. Then [KKLV89, Proposition 2.4] gives us $n_{i} \geqslant 1$ and linearizations in the sense of [MFK94, Definition 1.6]:

$$
\Phi_{i}: \alpha^{*} \mathcal{O}_{X}\left(n_{i} D_{i}\right) \rightarrow \operatorname{pr}_{X}^{*} \mathcal{O}_{X}\left(n_{i} D_{i}\right)
$$

Let $\Gamma^{\prime} \subset \Gamma(\Lambda)$ be the subgroup generated by the $n_{i} D_{i}$. Then, via tensoring the $\Phi_{i}$, we obtain for each $D \in \Gamma^{\prime} \cap \Lambda$ an isomorphism $\alpha^{*} \mathcal{A}_{D} \rightarrow \operatorname{pr}_{X}^{*} \mathcal{A}_{D}$. These maps are compatible with the multiplicative structures of $\alpha^{*} \mathcal{A}$ and $\operatorname{pr}_{X}^{*} \mathcal{A}$, and hence fit together to a linearization of $\Gamma^{\prime} \cap \Lambda$.

The second existence statement provides canonical G-linearizations. As usual, we say that a Weil divisor $D=\sum n_{E} E$ is $G$-invariant if $n_{g \cdot E}=n_{E}$ holds for any prime divisor $E$. The support of a $G$-invariant Weil divisor is $G$-invariant, whereas its components may be permuted.

Moreover, we have to consider pullbacks of $\mathcal{A}$ under dominant maps $p: Z \rightarrow X$, where $Z$ is normal. If the inverse image $p^{-1}\left(X^{\prime}\right)$ of the smooth locus $X^{\prime} \subset X$ has a complement of codimension at least two in $Z$, then the pullback $\operatorname{CDiv}\left(X^{\prime}\right) \rightarrow \operatorname{CDiv}\left(p^{-1}\left(X^{\prime}\right)\right)$ induces a map $p^{*}: \operatorname{WDiv}(X) \rightarrow$ $\operatorname{WDiv}(Z)$, and we obtain

$$
p^{*} \mathcal{A}=\bigoplus_{D \in \Lambda} \mathcal{O}_{Z}\left(p^{*} D\right)
$$

Proposition 1.7. Let $\Lambda$ consist of $G$-invariant divisors. Then there is a canonical $G$-linearization

$$
\alpha^{*} \mathcal{A}=\bigoplus_{D \in \Lambda} \mathcal{O}_{G \times X}\left(\alpha^{*} D\right)=\bigoplus_{D \in \Lambda} \mathcal{O}_{G \times X}\left(\operatorname{pr}_{X}^{*} D\right)=\operatorname{pr}_{X}^{*} \mathcal{A} .
$$

The induced $G$-sheaf structure on $\mathcal{A}$ is given by the usual action of $G$ on the function field $\mathbb{K}(X)$ via $g \cdot f(x)=f\left(g^{-1} \cdot x\right)$.

Proof. We have to show that any $G$-invariant Weil divisor $D=\sum n_{E} E$ satisfies $\alpha^{*} D=\operatorname{pr}_{X}^{*} D$. For this, we consider the isomorphism

$$
\beta: G \times X \rightarrow G \times X, \quad(g, x) \mapsto\left(g, g^{-1} \cdot x\right) .
$$

Then we have $\beta^{*} \operatorname{pr}_{X}^{*} D=\operatorname{pr}_{X}^{*} D$, and $\operatorname{pr}_{X}^{*} D=\beta^{*} \alpha^{*} D$. Since $\beta^{*}$ has an inverse, the assertion follows.

We turn to uniqueness properties of $G$-linearizations. Let Char $(G)$ denote the group of characters of $G$, i.e. the group of all homomorphisms $G \rightarrow \mathbb{K}^{*}$. For groups $G$ with few characters, we have the following two statements (compare [MFK94, Proposition 1.4] and [Hau01, Proposition 1.5]).

Proposition 1.8. Let $X$ be separated, and let $\Lambda \subset \operatorname{WDiv}(X)$ be a polyhedral semigroup.

i) If $\operatorname{Char}(G)$ is trivial and $G$ is connected, then any two $G$-linearizations of $\Lambda$ coincide.

ii) If $\operatorname{Char}(G)$ is finite and $\mathcal{O}^{*}(X)=\mathbb{K}^{*}$ holds, then any two G-linearizations of $\Lambda$ induce the same $G$-linearization on some $\Lambda^{\prime} \subset \Lambda$ of finite index. 


\section{J. Hausen}

Proof. Again by normality, it suffices to treat the problem over the smooth locus. Then $q: \widetilde{X} \rightarrow$ $X$ is locally trivial with toric fibres, having $S=\operatorname{Spec}(\mathbb{K}[\Gamma(\Lambda)])$ as their big torus. Given two $G$-linearizations of $\Lambda$, we denote the two corresponding $G$-actions on $\widetilde{X}$ by $g \cdot z$ and $g * z$. Consider the morphism

$$
\psi: G \times \widetilde{X} \rightarrow \widetilde{X}, \quad z \mapsto g^{-1} * g \cdot z .
$$

For fixed $g$, the map $z \mapsto \psi(g, z)$ is an $S$-equivariant bundle automorphism. Hence, on each fibre it is multiplication with an element of the torus $S$. Consequently, there is a morphism $\eta: G \times X \rightarrow S$ such that $\psi$ is of the form

$$
\psi(g, z)=\eta(g, q(z)) \cdot z .
$$

In the setting of part i, Rosenlicht's Lemma [FI73, Lemma 2.1] yields a decomposition $\eta(g, z)=$ $\chi(g) \beta(q(z))$ with a regular homomorphism $\chi: G \rightarrow S$ and a morphism $\beta: X \rightarrow S$. Since we assumed $G$ to have only trivial characters, we can conclude that $\psi$ is the identity map.

If we are in the situation of part ii, then $\mathcal{O}^{*}(X)=\mathbb{K}^{*}$ implies that $\psi(g, z)=\chi(g) \cdot z$ holds with a regular homomorphism $\chi: G \rightarrow S$. Hence, after dividing $\widetilde{X}$ by the finite subgroup $\chi(G) \subset S$, the two induced $G$-actions coincide. But this process means replacing $\Lambda$ with a subsemigroup of finite index.

Let us remark that there are simple examples showing that for nonconnected $G$, one cannot omit the assumption $\mathcal{O}^{*}(X)=\mathbb{K}^{*}$ in the second statement.

\section{The ample locus}

We introduce the Cartier locus and the ample locus of a polyhedral semigroup of Weil divisors, and study its behaviour in the case of $G$-linearized semigroups. The considerations of this section prepare the proofs of the various Hilbert-Mumford type theorems given later.

Unless otherwise stated, $X$ denotes in this section an irreducible normal prevariety. Given a polyhedral semigroup $\Lambda \subset \operatorname{WDiv}(X)$, let $\mathcal{A}$ denote the associated $\Lambda$-graded $\mathcal{O}_{X}$-algebra. For a homogeneous local section $f \in \mathcal{A}_{D}(U)$, we define its zero set to be

$$
Z(f):=\operatorname{Supp}\left(\operatorname{div}(f)+\left.D\right|_{U}\right) .
$$

Definition 2.1. Let $\Lambda \subset \operatorname{WDiv}(X)$ be a polyhedral semigroup with associated $\Lambda$-graded $\mathcal{O}_{X}$-algebra $\mathcal{A}$.

i) The Cartier locus of $\Lambda$ is the set of all points $x \in X$ such that every $D \in \Lambda$ is Cartier near $x$.

ii) The ample locus of $\Lambda$ is the set of all $x \in X$ admitting an affine neighbourhood $X \backslash Z(f)$ with a homogeneous section $f \in \mathcal{A}(X)$ such that $X \backslash Z(f)$ is contained in the Cartier locus of $\Lambda$.

We shall speak of an ample semigroup $\Lambda \subset \operatorname{WDiv}(X)$ if the ample locus of $\Lambda$ equals $X$. Thus, ample semigroups consist by definition of Cartier divisors. The relations to the usual ampleness concepts [GD61, Bor63, BGI71] are the following; recall that $X$ is said to be divisorial if every $x \in X$ has an affine neighbourhood $X \backslash \operatorname{Supp}(E)$ with an effective Cartier divisor $E$ on $X$.

Remark 2.2.

i) A polyhedral semigroup of the form $\Lambda=\mathbb{N} D$ is ample if and only if $D$ is an ample Cartier divisor in the usual sense.

ii) An irreducible normal prevariety is divisorial if and only if it admits an ample group of Cartier divisors. 


\section{GIT BASED ON WEIL DIVISORS}

Let us explain the geometric meaning of the ample locus of a polyhedral semigroup $\Lambda \subset \operatorname{CDiv}(X)$ in terms of the corresponding toric bundle $q: \widetilde{X} \rightarrow X$. Recall from Section 1 that $\widetilde{X}$ comes along with an action of the torus $S=\operatorname{Spec}(\mathbb{K}[\Gamma(\Lambda)])$, and that the set $\widehat{X} \subset \widetilde{X}$ of free $S$-orbits is an $S$-principal bundle over $X$.

Proposition 2.3. Let $\Lambda \subset \operatorname{CDiv}(X)$ be a polyhedral semigroup with associated toric bundle $q: \widetilde{X} \rightarrow X$ and ample locus $U \subset X$. Then $q^{-1}(U) \cap \widehat{X}$ is quasiaffine.

Proof. Consider the subgroup $\Gamma(\Lambda) \subset \operatorname{CDiv}(X)$ generated by $\Lambda$, and denote the associated graded $\mathcal{O}_{X}$-algebra by $\mathcal{B}$. Then $\widehat{X}$ equals $\operatorname{Spec}(\mathcal{B})$, and for any homogeneous $f \in \mathcal{B}(X)$, its zero set as a function on $\widehat{X}$ is equal to the inverse image $q^{-1}(Z(f)) \cap \widehat{X}$. Consequently, the set $q^{-1}(U) \cap \widehat{X}$ is covered by affine open subsets of the form $\widehat{X}_{f}$ with $f \in \mathcal{O}(\widehat{X})$. This gives the assertion.

We turn to the equivariant setting. Let $G$ be a linear algebraic group, and suppose that $G$ acts on the normal prevariety $X$. A first observation is that the zero set $Z(f)$ of a homogeneous section $f$ behaves natural with respect to the $G$-sheaf structure of Proposition 1.4 arising from a $G$-linearization.

Lemma 2.4. Let $\Lambda \subset \operatorname{WDiv}(X)$ be a $G$-linearized polyhedral semigroup, and let $f \in \mathcal{A}_{D}(U)$ be a local section of the associated graded $\mathcal{O}_{X}$-algebra $\mathcal{A}$. Then we have $Z(g \cdot f)=g \cdot Z(f)$ for any $g \in G$.

Proof. By normality of $X$, we may assume that $U$ is smooth. The problem being local, we may moreover assume that $D$ is principal on $U$, say $D=-\operatorname{div}(h)$. Then the section $f$ is of the form $f=f^{\prime} h$ with a regular function $f^{\prime}$, and $Z(f)$ is just the zero set $Z\left(f^{\prime}\right)$ of $f^{\prime}$. Translating with $g \in G$ gives

$$
Z(g \cdot f)=Z\left(g \cdot f^{\prime} g \cdot h\right)=Z\left(g \cdot f^{\prime}\right) \cup Z(g \cdot h) .
$$

Since $h$ is a generator of $\mathcal{A}(U)$, the translate $g \cdot h$ is a generator of $\mathcal{A}(g \cdot U)$. This means that $Z(g \cdot h)$ is empty. By definition of the $G$-sheaf structure, $G$ acts canonically on the structure sheaf $\mathcal{O}_{X}$, which means that $g \cdot f^{\prime}(x)$ equals $f^{\prime}\left(g^{-1} \cdot x\right)$. This implies $Z\left(g \cdot f^{\prime}\right)=g \cdot Z\left(f^{\prime}\right)$, and the assertion follows.

Proposition 2.5. Let $\Lambda \subset \operatorname{WDiv}(X)$ be a $G$-linearized polyhedral semigroup. Then the Cartier locus and the ample locus of $\Lambda$ are $G$-invariant.

Proof. Let $\mathcal{A}$ denote the graded $\mathcal{O}_{X}$-algebra corresponding to $\Lambda$. The Cartier locus of $\Lambda$ is the set of all points $x \in X$ such that for any $D \in \Lambda$ the stalk $\mathcal{A}_{D, x}$ is generated by a single element. Thus, using the $G$-sheaf structure of $\mathcal{A}$, we obtain that the Cartier locus is $G$-invariant. Invariance of the ample locus is then a simple consequence of Lemma 2.4 .

As a direct application, we extend a fundamental observation of Sumihiro on actions of connected linear algebraic groups $G$ on normal varieties $X$ (see [Sum74, Lemma 8] and [Sum75, Theorem 3.8]): Every point $x \in X$ admits a $G$-invariant quasiprojective open neighbourhood. Our methods give more generally the following proposition.

Proposition 2.6. Let $G$ be a connected linear algebraic group, let $X$ be a normal $G$-variety, and let $U \subset X$ be an open subset.

i) If $U$ is quasiprojective, then $G \cdot U$ is quasiprojective.

ii) If $U$ is divisorial, then $G \cdot U$ is divisorial.

In particular, the maximal quasiprojective and the maximal divisorial open subsets of $X$ are $G$-invariant. 


\section{J. HAUSEN}

If $X$ admits a normal completion for which the factor group of Weil divisors modulo $\mathbb{Q}$-Cartier divisors is of finite rank, then [Wlo99, Theorem A] says that $X$ has only finitely many maximal open quasiprojective subvarieties. In particular, then Proposition 2.6, part i even holds with any connected algebraic group $G$ (see [Wlo99, Theorem D]). A special case of the second statement is proved in [ACH02, Lemma 1.7].

Lemma 2.7. Let $X$ be a normal variety, $D^{\prime}$ a Weil divisor on some open $U \subset X$, and $f_{1}^{\prime}, \ldots, f_{r}^{\prime}$ sections of $D^{\prime}$ with $U \backslash Z\left(f_{i}^{\prime}\right)$ affine. Then there is a Weil divisor $D$ on $X$ allowing global sections $f_{1}, \ldots, f_{r}$ such that

$$
\left.D\right|_{U}=D^{\prime},\left.\quad f_{i}\right|_{U}=f_{i}^{\prime}, \quad X \backslash Z\left(f_{i}\right)=U \backslash Z\left(f_{i}^{\prime}\right)
$$

Moreover, if $U$ and $D^{\prime}$ are invariant with respect to a given algebraic group action on $X$, then one can also choose $D$ to be so.

Proof. Let $D_{1}, \ldots, D_{s}$ be the prime divisors contained in $X \backslash U$. Since the complement of $U \backslash Z\left(f_{i}^{\prime}\right)$ in $X$ is of pure codimension one, we have

$$
U \backslash Z\left(f_{i}^{\prime}\right)=X \backslash\left(D_{1} \cup \cdots \cup D_{s} \cup \overline{Z\left(f_{i}^{\prime}\right)}\right) .
$$

Consequently, by closing the components of $D^{\prime}$ and adding a suitably big multiple of $D_{1}+\cdots+D_{s}$, we obtain the desired Weil divisor $D$ on $X$.

Proof of Proposition 2.6. For part i, we choose a $D^{\prime} \in \operatorname{CDiv}(U)$ allowing sections $f_{1}^{\prime}, \ldots, f_{r}^{\prime}$ such that the sets $U \backslash Z\left(f_{i}^{\prime}\right)$ are affine and cover $U$. Similarly, for part ii, we find $D_{1}^{\prime}, \ldots D_{r}^{\prime} \in \operatorname{CDiv}(U)$ allowing sections $f_{1}^{\prime}, \ldots, f_{r}^{\prime}$ such that the sets $U \backslash Z\left(f_{i}^{\prime}\right)$ are affine and cover $U$.

Use Lemma 2.7 to extend $D^{\prime}$ (respectively the $D_{i}^{\prime}$ ) to Weil divisors $D$ (respectively $D_{i}$ ) on $X$ such that the $f_{i}^{\prime}$ extend to global sections $f_{i}$ over $X$ and satisfy $X \backslash Z\left(f_{i}\right)=U \backslash Z\left(f_{i}^{\prime}\right)$. Let $\Lambda$ be the semigroup generated by $D$ (respectively the subgroup generated by the $D_{i}$ ). Then, in both cases $U$ is contained in the ample locus of the extension $\Lambda$.

Now, passing to subsemigroups of finite index does not shrink the ample locus. Hence, we can use Proposition 1.6, and endow $\Lambda$ with a $G$-linearization. The assertion then follows from $G$-invariance of the ample locus of $\Lambda$ and the fact that quasiprojectivity as well as divisoriality transfer to open subvarieties.

We conclude this section with an equivariant and refined version of Proposition 2.3; again, we consider the subset $\widehat{X} \subset \widetilde{X}$ of free orbits of the torus $S=\operatorname{Spec}(\mathbb{K}[\Gamma(\Lambda)])$.

Proposition 2.8. Let $\Lambda \subset \operatorname{CDiv}(X)$ be a $G$-linearized polyhedral semigroup with associated toric bundle $q: \widetilde{X} \rightarrow X$. Let $U \subset X$ be the ample locus of $\Lambda$, and set $\widehat{U}:=\widehat{X} \cap q^{-1}(U)$. Then there is a $(G \times S)$-equivariant open embedding $\widehat{U} \rightarrow Z$ into an affine $(G \times S)$-variety $Z$. Moreover, the following hold.

i) One can achieve that the image of the pullback map $\mathcal{O}(Z) \rightarrow \mathcal{O}(\widehat{U})$ is contained in $\mathcal{O}(\widetilde{X})$.

ii) Given $f_{1}, \ldots, f_{k} \in \mathcal{A}(X)$ as in Definition 2.1 with $\widetilde{X}_{f_{i}} \subset \widehat{X}$, one can achieve that each $f_{i}$ extends regularly to $Z$ and satisfies $\widehat{U}_{f_{i}}=Z_{f_{i}}$.

iii) For every $f \in \mathcal{O}(Z) \subset \mathcal{O}(\widetilde{X})$ with $\widetilde{X}_{f} \subset \widehat{X}$ and $\left.f\right|_{Z \backslash \widehat{U}}=0$, we have $Z_{f}=\widehat{X}_{f}$.

Proof. Let $f_{1}, \ldots, f_{k} \in \mathcal{A}(X)$ be as in part ii, and complement this collection by further homogeneous sections $f_{k+1}, \ldots, f_{r} \in \mathcal{A}(X)$ as in Definition 2.1 such that the affine sets $X_{i}:=X \backslash Z\left(f_{i}\right)$ cover the ample locus $U \subset X$. Then each $f_{i}$, regarded as a regular function on $\widetilde{X}$, vanishes outside the affine open set $\widetilde{X}_{i}:=q^{-1}\left(X_{i}\right)$ and has no zeroes inside $\widetilde{X}_{i} \cap \widehat{X}$.

For each $i$, we choose finitely many homogeneous functions $h_{i j} \in \mathcal{O}(\widetilde{X})$ such that the affine algebra $\mathcal{O}(\tilde{X})_{f_{i}}$ is generated by functions $h_{i j} / f_{i}^{l_{i j}}$. Since the $G$-representation on $\mathcal{O}(\tilde{X})$ is rational, 


\section{GIT BASED ON WEIL DIVISORS}

we find finite-dimensional graded $G$-modules $M_{i}, M_{i j} \subset \mathcal{O}(\widetilde{X})$ such that $f_{i} \in M_{i}$ and $h_{i j} \in M_{i j}$ holds.

Let $R \subset \mathcal{O}(\widetilde{X})$ denote the subalgebra generated by the elements of the $M_{i}$ and the $M_{i j}$. Then $R$ is graded, $G$-invariant, and hence defines an affine $(G \times S)$-variety $Z:=\operatorname{Spec}(R)$. Note that $Z_{f_{i}}=\widetilde{X}_{f_{i}}$ holds. This gives $\widehat{U} \subset Z$ and part ii. Moreover, we obtain part iii by covering $\widetilde{X}_{f}$ and $Z_{f}$ with the affine sets $\widetilde{X}_{f_{i} f}=Z_{f_{i} f}$.

\section{Construction of quotients}

In this section, $G$ is a reductive group, and $X$ is a normal $G$-variety. We describe the $G$-invariant open subsets $U \subset X$ admitting a good quotient with a quasiprojective or a divisorial good quotient space. First recall the precise definition of a good quotient (compare [MFK94, p. 38] and [Ses72, Definition 1.5]).

Definition 3.1. A good quotient for a $G$-prevariety $X$ is an affine $G$-invariant morphism $p: X \rightarrow Y$ such that the canonical map $\mathcal{O}_{Y} \rightarrow p_{*}\left(\mathcal{O}_{X}\right)^{G}$ is an isomorphism. A good quotient is called geometric if its fibres are precisely the $G$-orbits.

In our setting, a separated $G$-variety may have a good quotient with a nonseparated quotient space. If a good quotient $X \rightarrow Y$ exists for a $G$-variety $X$, then it is categorical, i.e. any $G$-invariant morphism $X \rightarrow Z$ factors uniquely through $X \rightarrow Y$. In particular, good quotient spaces are unique up to isomorphism. As usual, we write $X \rightarrow X / / G$ for a good and $X \rightarrow X / G$ for a geometric quotient.

In general, the $G$-variety $X$ itself need not admit a good quotient, but there frequently exist many $G$-invariant open subsets $U \subset X$ with a good quotient. Following [BB02], we say that a subset $V$ of an open $G$-invariant subset $U \subset X$ with good quotient $p: U \rightarrow U / / G$ is $G$-saturated in $U$ if $V=p^{-1}(p(V))$ holds.

We begin with the construction of quasiprojective good quotient spaces. Fix a Weil divisor $D$ on $X$, and a $G$-linearization of the semigroup $\Lambda:=\mathbb{N} D$; we shall speak in the sequel of the $G$-linearized Weil divisor $D$. Recall from Proposition 1.4 that there is an induced $G$-representation on the global sections $\mathcal{A}(X)$ of the associated $\Lambda$-graded $\mathcal{O}_{X}$-algebra $\mathcal{A}$.

Definition 3.2. We call a point $x \in X$ semistable if there is an integer $n>0$ and a $G$-invariant $f \in \mathcal{A}_{n D}(X)$ such that $X \backslash Z(f)$ is a affine neighbourhood of $x$ and $D$ is Cartier on $X \backslash Z(f)$.

Following Mumford's notation, we denote the set of semistable points of a $G$-linearized Weil divisor $D$ on $X$ by $X^{\mathrm{ss}}(D)$, or by $X^{\mathrm{ss}}(D, G)$ if we want to specify the group $G$. Our concept of semistability yields all open subsets admitting a quasiprojective good quotient space.

Theorem 3.3. Let a reductive group $G$ act on a normal variety $X$.

i) For any $G$-linearized Weil divisor $D$ on $X$, there is a good quotient $X^{\mathrm{ss}}(D) \rightarrow X^{\mathrm{ss}}(D) / / G$ with a quasiprojective variety $X^{\mathrm{ss}}(D) / / G$.

ii) If $U \subset X$ is open, $G$-invariant, and has a good quotient $U \rightarrow U / / G$ with $U / / G$ quasiprojective, then $U$ is a $G$-saturated subset of the set $X^{\mathrm{ss}}(D)$ of semistable points of a canonically $G$-linearized Weil divisor $D$.

Proof. For part i, we can follow the lines of [MFK94, Theorem 1.10]: Choose G-invariant homogeneous sections $f_{1}, \ldots, f_{r} \in \mathcal{A}(X)$ as in Definition 3.2 such that $X^{\mathrm{ss}}(D)$ is covered by the sets $X_{i}:=X \backslash Z\left(f_{i}\right)$. Replacing the $f_{i}$ with suitable powers, we may assume that all of them have the same degree. Consider the good quotients:

$$
p_{i}: X_{i} \rightarrow X_{i} / / G=\operatorname{Spec}\left(\mathcal{O}\left(X_{i}\right)^{G}\right) .
$$




\section{J. HAUSEN}

Each $X_{i} \backslash X_{j}$ is the zero set of the $G$-invariant regular function $f_{j} / f_{i}$. Thus $X_{i} \cap X_{j}$ is saturated with respect to the quotient map $p_{i}: X_{i} \rightarrow X_{i} / / G$. It follows that the $p_{i}$ glue together to a good quotient $p: X^{\mathrm{ss}}(D) \rightarrow X^{\mathrm{ss}}(D) / / G$. Moreover, for fixed $i_{0}$, the $f_{i_{0}} / f_{i}$ are local equations for an ample divisor on $X^{\mathrm{ss}}(D) / / G$.

To prove part ii, let $Y:=U / / G$, and let $p: U \rightarrow Y$ be the quotient map. Choose an ample divisor $E$ on $Y$ allowing global sections $h_{1}, \ldots, h_{r}$ such that the sets $Y \backslash Z\left(h_{i}\right)$ form an affine cover of $Y$. Consider the pullback data $D^{\prime}:=p^{*} E$ and $f_{i}^{\prime}:=p^{*}\left(h_{i}\right)$. Then Lemma 2.7 provides a $G$-invariant Weil divisor $D$ on $X$ extending $D^{\prime}$ and sections $f_{i}$ extending $f_{i}^{\prime}$ such that

$$
X \backslash Z\left(f_{i}\right)=U \backslash Z\left(f_{i}^{\prime}\right)=p^{-1}\left(Y \backslash Z\left(h_{i}\right)\right) .
$$

Let $\mathcal{A}$ be the graded $\mathcal{O}_{X}$-algebra associated to $D$, and consider the canonical $G$-linearization of $D$ provided by Proposition 1.7. Then the sections $f_{i} \in \mathcal{A}(X)$ are $G$-invariant, and satisfy the conditions of Definition 3.2. It follows that $U$ is a saturated subset of $X^{\mathrm{ss}}(D)$.

For the construction of divisorial quotient spaces, we work with finitely generated subgroups $\Lambda \subset \mathrm{WDiv}(X)$; these are in particular polyhedral semigroups. Fix such a subgroup $\Lambda \subset \operatorname{WDiv}(X)$, and a $G$-linearization of $\Lambda$ as introduced in Section 1. Again, we have an induced $G$-representation on the global sections $\mathcal{A}(X)$ of the associated $\Lambda$-graded $\mathcal{O}_{X}$-algebra $\mathcal{A}$.

Definition 3.4. We call a point $x \in X$ semistable if $x$ has an affine neighbourhood $U=X \backslash Z(f)$ with some $G$-invariant homogeneous $f \in \mathcal{A}(X)$ such that all $D \in \Lambda$ are Cartier on $U$, and the $D \in \Lambda$ admitting a $G$-invariant invertible $h \in \mathcal{A}_{D}(U)$ form a subgroup of finite index in $\Lambda$.

As before, the set of semistable points is denoted by $X^{\mathrm{ss}}(\Lambda)$, or $X^{\mathrm{ss}}(\Lambda, G)$ if we want to specify the group $G$. Note that for $G$-linearized groups of Cartier divisors we retrieve the notion of semistability introduced in [Hau01, Definition 2.1]. We obtain the following generalizations of [Hau01, Theorems 3.1 and 4.1].

TheOREM 3.5. Let a reductive group $G$ act on a normal variety $X$.

i) For any $G$-linearized group $\Lambda \subset \operatorname{WDiv}(X)$, there is a good quotient $X^{\mathrm{ss}}(\Lambda) \rightarrow X^{\mathrm{ss}}(\Lambda) / / G$ with a divisorial prevariety $X^{\mathrm{ss}}(D) / / G$.

ii) If $U \subset X$ is open, $G$-invariant, and admits a good quotient $U \rightarrow U / / G$ with $U / / G$ divisorial, then $U$ is a $G$-saturated subset of the set $X^{\mathrm{ss}}(\Lambda)$ of semistable points of a canonically $G$-linearized group $\Lambda \subset \operatorname{WDiv}(X)$.

Proof. To prove part i, consider the Cartier locus $X_{0} \subset X$ of $\Lambda$. By Proposition 2.5, the set $X_{0}$ is $G$-invariant. Since $X$ is normal, $X \backslash X_{0}$ is of codimension at least two in $X$. Hence $X_{0}^{\text {ss }}(\Lambda)$ equals $X^{\mathrm{ss}}(\Lambda)$, and we may assume that $\Lambda$ consists of Cartier divisors. But then [Hau01, Theorem 3.1] gives the assertion.

The proof of part ii is analogous to that of [Hau01, Theorem 4.1]. Using divisoriality of $Y:=U / / G$ and [Hau01, Lemma 4.3], we find effective $E_{1}, \ldots, E_{r} \in \operatorname{CDiv}(Y)$ and global sections $h_{i j}$ of the $E_{i}$ such that the sets $V_{i j}:=Y \backslash Z\left(h_{i j}\right)$ form an affine cover of $Y$, and every $E_{k}$ admits an invertible section $h_{i j k}$ over $V_{i j}$.

Let $p: U \rightarrow Y$ be the quotient map. Lemma 2.7 provides invariant Weil divisors $D_{i}$ on $X$ admitting global sections $f_{i j}$ such that with $U_{i j}:=p^{-1}\left(V_{i j}\right)$ we have

$$
\left.D_{i}\right|_{U}=p^{*} E_{i},\left.\quad f_{i j}\right|_{U_{i j}}=p^{*}\left(h_{i j}\right), \quad X \backslash Z\left(f_{i j}\right)=p^{-1}\left(V_{i j}\right) .
$$

By Proposition 1.7, the group $\Lambda \subset \operatorname{WDiv}(X)$ generated by the $D_{i}$ is canonically $G$-linearized. The sections $f_{i j}$ and $p^{*}\left(h_{i j k}\right)$ serve to verify $U \subset X^{\mathrm{ss}}(\Lambda)$. Since each $U_{i j}$ is $G$-saturated in $X^{\mathrm{ss}}(\Lambda)$, the same holds for $U$. 


\section{GIT BASED ON WEIL DIVISORS}

We conclude the section with an example, showing that in the singular case Mumford's method and the generalization given in [Hau01] need no longer provide all open subsets with quasiprojective or divisorial quotient spaces. Consider the cone $X$ over the image of $\mathbb{P}_{1} \times \mathbb{P}_{1}$ in $\mathbb{P}_{3}$ under the Segre embedding, i.e.

$$
X=V\left(\mathbb{K}^{4} ; z_{1} z_{3}-z_{2} z_{4}\right)
$$

Then $X$ is a normal variety having precisely one singular point. Let $U:=X_{z_{2}} \cup X_{z_{4}}$ be the set of points having nonvanishing second or fourth coordinate. We consider the following action of the two-dimensional torus $T:=\mathbb{K}^{*} \times \mathbb{K}^{*}$ on $X$ :

$$
t \cdot x:=\left(t_{1}^{2} x_{1}, t_{1} t_{2}^{2} x_{2}, t_{1} t_{2} x_{3}, t_{1}^{2} t_{2}^{-1} x_{4}\right) .
$$

Proposition 3.6. The set $U \subset X$ has a geometric quotient $U \rightarrow U / T$ with $U / T \cong \mathbb{P}_{1}$, but $U$ is not the set of semistable points of a T-linearized line bundle on $X$ in the sense of [MFK94, Definition 1.7].

Proof. The most convenient way is to view $X$ as a toric variety, and to work in the language of lattice fans (see [Ful93] for the basic notions). As a toric variety, $X$ corresponds to the lattice cone $\sigma$ in $\mathbb{Z}^{3}$ generated by the vectors

$$
v_{1}:=(1,0,0), \quad v_{2}:=(0,1,0), \quad v_{3}:=(0,1,1), \quad v_{4}:=(1,0,1) .
$$

The big torus of $X$ is $T_{X}=\left(\mathbb{K}^{*}\right)^{3}$. The torus $T$ acts on $X$ by $(t, x) \mapsto \varphi(t) \cdot x$, where $\varphi: T \rightarrow T_{X}$ is the homomorphism of tori corresponding to the linear map

$$
\mathbb{Z}^{2} \rightarrow \mathbb{Z}^{3}, \quad(1,0) \mapsto(2,1,1), \quad(0,1) \mapsto(0,2,1) .
$$

Our open set $U \subset X$ is a union of three $T_{X}$-orbits: the big $T_{X}$-orbit, and the two two-dimensional $T_{X}$-orbits corresponding to the rays $\varrho_{1}:=\mathbb{Q}_{\geqslant 0} v_{1}$ and $\varrho_{3}:=\mathbb{Q}_{\geqslant 0} v_{3}$ of the cone $\sigma$. The fan theoretical criterion [Ham00, Theorem 5.1] tells us that there is a geometric quotient for the action of $T$ on $U$; namely the toric morphism $p: U \rightarrow \mathbb{P}_{1}$ defined by the linear map

$$
P: \mathbb{Z}^{3} \rightarrow \mathbb{Z}, \quad\left(w_{1}, w_{2}, w_{3}\right) \mapsto w_{1}+2 w_{2}-4 w_{3} .
$$

We show now that there is no $T$-linearized line bundle on $X$ having $U$ as its set of semistable points. First note that, as an affine toric variety, $X$ has trivial Picard group. Thus we only have to consider $T$-linearizations of the trivial bundle. Since $\mathcal{O}^{*}(X)=\mathbb{K}^{*}$ holds, each such linearization is given by a character $\chi$ of $T$ :

$$
t \cdot(x, z)=(t \cdot x, \chi(t) z) .
$$

Consequently, in view of [MFK94, Definition 1.7], we have to show that $U$ is not a union of sets $X_{f}$, for a collection of functions $f \in \mathcal{O}(X)$ that are $T$-homogeneous with respect to a common character of the torus $T$.

Now, any $T$-homogeneous regular function on $X$ is a sum of $T$-homogeneous character functions $\chi^{u} \in \mathcal{O}(X)$, where $u=\left(u_{1}, u_{2}, u_{3}\right)$ is a lattice vector of the dual cone $\sigma^{\vee}$ of $\sigma$. Recall that $u \in \sigma^{\vee}$ means that the linear form $u$ is nonnegative on $\sigma$, i.e. we have

$$
u_{1} \geqslant 0, \quad u_{2} \geqslant 0, \quad u_{2}+u_{3} \geqslant 0, \quad u_{1}+u_{3} \geqslant 0 .
$$

For such a character function $\chi^{u} \in \mathcal{O}(X)$, we can determine its weight with respect to $T$ by applying the dual of the embedding $\mathbb{Z}^{2} \rightarrow \mathbb{Z}^{3}$ to the vector $u$. Thus, $\chi^{u}$ is $T$-homogeneous with respect to the character of $T$ corresponding to the lattice vector

$$
\left(2 u_{1}+u_{2}+u_{3}, 2 u_{2}+u_{3}\right) .
$$




\section{J. HAUSEN}

The conditions that a character function $\chi^{u} \in \mathcal{O}(X)$ does not vanish along the orbit $T_{X} \cdot x_{i}$ corresponding to one of the rays $\varrho_{i}$ are $u_{1}=0$ for nonvanishing along $T_{X} \cdot x_{1}$, and $u_{3}=-u_{2}$ for nonvanishing along $T_{X} \cdot x_{3}$.

Suppose that $\chi^{u} \in \mathcal{O}(X)$ does not vanish along $T_{X} \cdot x_{1}$ and that $\chi^{\widetilde{u}} \in \mathcal{O}(X)$ does not vanish along $T_{X} \cdot x_{3}$. Then their respective $T$-weights are given by the vectors

$$
\left(u_{2}+u_{3}, 2 u_{2}+u_{3}\right), \quad\left(2 \widetilde{u}_{1}, \widetilde{u}_{2}\right) .
$$

If both are $T$-homogeneous with respect to the same character, then we must have $2 \widetilde{u}_{1} \leqslant \widetilde{u}_{2}$. But then nonvanishing along $T_{X} \cdot x_{3}$ and the last regularity condition imply $\widetilde{u}=0$.

In conclusion, we obtain that only the trivial character of $T$ admits homogeneous functions that do not vanish along $T_{X} \cdot x_{1}$ and functions that do not vanish along $T_{X} \cdot x_{3}$. Since $T$ acts with an attractive fixed point on $X$, this means that we cannot obtain $U$ as a union of sets $X_{f}$ as needed.

\section{First Hilbert-Mumford type statements}

We come to the first Hilbert-Mumford type result of the paper. It allows us to express the set of $G$-semistable points in terms of the $T$-semistable points for a maximal torus $T \subset G$. In the case of an ample divisor $D$ on a projective $G$-variety, the first assertion of our result is equivalent to [MFK94, Theorem 2.1].

Theorem 4.1. Let a reductive group $G$ act on a normal variety $X$, and let $T \subset G$ be a maximal torus.

i) Let $D$ be a $G$-linearized Weil divisor on $X$. Then we have

$$
X^{\mathrm{ss}}(D, G)=\bigcap_{g \in G} g \cdot X^{\mathrm{ss}}(D, T) .
$$

ii) Let $\Lambda \subset \operatorname{WDiv}(X)$ be a $G$-linearized subgroup. Then we have

$$
X^{\mathrm{ss}}(\Lambda, G)=\bigcap_{g \in G} g \cdot X^{\mathrm{ss}}(\Lambda, T) .
$$

The proof (presented after Lemma 4.5) relies on a geometric analysis of instability; it makes repeated use of the classical Hilbert-Mumford Theorem (see for example [Bir71, Theorem 4.2]).

Theorem 4.2. Let a reductive group $G$ act on an affine variety $Z$, let $z \in Z$, and let $Y \subset$ $\overline{G \cdot z}$ be a $G$-invariant closed subset. Then there is a one-parameter subgroup $\lambda: \mathbb{K}^{*} \rightarrow G$ with $\lim _{t \rightarrow 0} \lambda(t) \cdot z \in Y$.

The basic preparatory steps concern the following situation: $G$ is a reductive group, $Z$ is an affine $G$-variety, and $T \subset G$ is a maximal torus. Then we have good quotients

$$
p_{T}: Z \rightarrow Z / / T, \quad p_{G}: Z \rightarrow Z / / G .
$$

Lemma 4.3. Let $A \subset Z$ be $G$-invariant and closed, and let $z \in p_{G}^{-1}\left(p_{G}(A)\right)$. Then there is a $g \in G$ with $g \cdot z \in p_{T}^{-1}\left(p_{T}(A)\right)$.

Proof. Since $p_{G}: Z \rightarrow Z / / G$ separates disjoint $G$-invariant closed sets, the closure of $G \cdot z$ intersects $A$. By Theorem 4.2, there is a maximal torus $S \subset G$ such that the closure of $S \cdot z$ intersects $A$. Choose a $g \in G$ with $T=g S g^{-1}$. Then the closure of $T \cdot g \cdot z$ intersects $A$. This implies $p_{T}(g \cdot z) \in p_{T}^{-1}\left(p_{T}(A)\right)$.

Suppose that in addition to the $G$-action there is an action of $\mathbb{K}^{*}$ on $Z$ such that these two actions commute. Then there are induced $\mathbb{K}^{*}$-actions on the quotient spaces $Z / / T$ and $Z / / G$ making 


\section{GIT BASED ON WEIL DIVISORS}

the respective quotient maps equivariant. Let $B_{T}^{0} \subset Z / / T$ and $B_{G}^{0} \subset Z / / G$ denote the fixed point sets of these $\mathbb{K}^{*}$-actions.

Lemma 4.4. Let $z \in Z$ with $p_{G}(z) \in B_{G}^{0}$. Then there is a $g \in G$ with $p_{T}(g \cdot z) \in B_{T}^{0}$.

Proof. Let $G \cdot z_{0}$ be the closed $G$-orbit in the fibre $p_{G}^{-1}\left(p_{G}(z)\right)$. If $z_{0}$ is a fixed point of the $\mathbb{K}^{*}$-action on $Z$, then the whole orbit $G \cdot z_{0}$ consists of $\mathbb{K}^{*}$-fixed points, and the assertion is a direct consequence of Theorem 4.2. So we may assume for this proof that the orbit $\mathbb{K}^{*} \cdot z_{0}$ is nontrivial.

By Theorem 4.2, there is a one-dimensional subtorus $S_{0} \subset G$ and a $g_{0} \in G$ such that $z_{0}$ lies in the closure of $S_{0} \cdot z^{\prime}$, where $z^{\prime}:=g_{0} \cdot z$. Note that, for any $t \in \mathbb{K}^{*}$, the point $t \cdot z_{0}$ lies in the closure of $S_{0} \cdot t \cdot z^{\prime}$. This implies in particular that any point of $\mathbb{K}^{*} \cdot z_{0}$ is fixed by $S_{0}$. Consequently, $S_{0}$ is a subgroup of the stabilizer $G_{0}$ of $\mathbb{K}^{*} \cdot z_{0}$.

Let $n \in \mathbb{N}$ denote the order of the isotropy group of $\mathbb{K}^{*}$ in $z_{0}$. Then the orbit maps $\mu: g \mapsto g \cdot z_{0}$ of $G_{0}$ and $\nu: t \mapsto t \cdot z_{0}$ of $\mathbb{K}^{*}$ give rise to a well defined morphism of linear algebraic groups:

$$
G_{0} \rightarrow \mathbb{K}^{*}, \quad g \mapsto\left(\nu^{-1}(\mu(g))\right)^{n} .
$$

Clearly, $S_{0}$ is contained in the kernel of this homomorphism. By general properties of linear algebraic groups, any maximal torus of $G_{0}$ is mapped onto $\mathbb{K}^{*}$ (see e.g. [Hum81, Corollary C, p. 136]). We choose a maximal torus $S_{1} \subset G_{0}$ such that $S_{1}$ contains $S_{0}$.

Let $S \subset G$ be a maximal torus with $S_{1} \subset S$. Then $z_{0}$ lies in the closure of $S \cdot z^{\prime}$. Moreover, $\mathbb{K}^{*} \cdot z_{0}$ is contained in $S \cdot z_{0}$. Writing $S=g_{1}^{-1} T g_{1}$ with a suitable $g_{1} \in G$, we obtain that $g_{1} \cdot z_{0}$ lies in the closure of $T \cdot g_{1} \cdot z^{\prime}$, and $\mathbb{K}^{*} \cdot g_{1} \cdot z_{0}$ is contained in $T \cdot g_{1} \cdot z_{0}$. Thus, $g:=g_{1} g_{0}$ is as wanted.

The next observation concerns limits with respect to the $\mathbb{K}^{*}$-action on the quotient spaces. For $H=T$ and $H=G$ we consider the sets:

$$
B_{H}^{-}:=\left\{y \in Z / / H ; \lim _{t \rightarrow \infty} t \cdot y \text { exists and differs from } y\right\} .
$$

Lemma 4.5. Let $z \in Z$ with $p_{G}(z) \in B_{G}^{-}$. Then there is a $g \in G$ such that $p_{T}(g \cdot z) \in B_{T}^{-}$holds.

Proof. Let $y_{0} \in Z / / G$ be the limit point of $p_{G}(z)$, and choose $z_{0} \in Z$ with $G \cdot z_{0}$ closed in $Z$ and $p_{G}\left(z_{0}\right)=y_{0}$. Note that $G \cdot z_{0}$ is $\mathbb{K}^{*}$-invariant. Consider the quotient $q: Z \rightarrow Z / / \mathbb{K}^{*}$. Then $G \cdot q\left(z_{0}\right)$ is contained in the closure of $G \cdot q(z)$, because $q\left(G \cdot z_{0}\right)$ is closed, and we have

$$
(Z / / G) / / \mathbb{K}^{*}=\left(Z / / \mathbb{K}^{*}\right) / / G .
$$

Thus, according to Theorem 4.2, there exist $g, g_{0} \in G$ such that $g_{0} \cdot q\left(z_{0}\right)$ lies in the closure of $T \cdot g \cdot q(z)$. We can conclude that, in $Z / / T$, the $\mathbb{K}^{*}$-orbit closures of the points $p_{T}(g \cdot z)$ and $p_{T}\left(g_{0} \cdot z_{0}\right)$ intersect nontrivially; this time we use

$$
(Z / / T) / / \mathbb{K}^{*}=\left(Z / / \mathbb{K}^{*}\right) / / T \text {. }
$$

Since we have a $\mathbb{K}^{*}$-equivariant map $Z / / T \rightarrow Z / / G$, and there is a $G$-invariant homogeneous function $f \in \mathcal{O}(Z)$ of negative weight with $f(z) \neq 0$ and $f\left(z_{0}\right)=0$, it follows that $p_{T}(g \cdot z)$ belongs to $B_{T}^{-}$.

Proof of Theorem 4.1. For part i, we may assume that $D$ is nontrivial. By Proposition 2.5, the Cartier locus $X_{0} \subset X$ of $\Lambda:=\mathbb{N} D$ is $G$-invariant. Moreover, by normality of $X$, the complement $X \backslash X_{0}$ is of codimension at least two in $X$. Consequently, $X_{0}^{\mathrm{ss}}(D, T)$ equals $X^{\mathrm{ss}}(D, T)$, and $X_{0}^{\mathrm{ss}}(D, G)$ equals $X^{\mathrm{ss}}(D, G)$. Thus we may assume for this proof that $X=X_{0}$ holds.

Let $\mathcal{A}$ be the graded $\mathcal{O}_{X}$-algebra associated to $\Lambda$. The associated $\widetilde{X}:=\operatorname{Spec}(\mathcal{A})$ is a line bundle over $X$, and the torus acting on $\widetilde{X}$ is $\mathbb{K}^{*}$. Consider the $G$-action on $\widetilde{X}$ provided by Proposition 1.3. 


\section{J. HAUSEN}

Removing the zero section gives the $\left(G \times \mathbb{K}^{*}\right)$-invariant open subvariety $\widehat{X} \subset \widetilde{X}$. Let $q: \widetilde{X} \rightarrow X$ be the canonical $G$-equivariant map, $U \subset X$ the ample locus of $D$, and $\widehat{U}:=q^{-1}(U) \cap \widehat{X}$.

Choose $T$-invariant homogeneous $f_{1}, \ldots, f_{r} \in \mathcal{A}(X)$ and $G$-invariant homogeneous $h_{1}, \ldots, h_{s} \in$ $\mathcal{A}(X)$ as in Definition 3.2 such that the sets $X \backslash Z\left(f_{i}\right)$ and $X \backslash Z\left(h_{j}\right)$ cover $X^{\mathrm{ss}}(D, T)$ and $X^{\mathrm{ss}}(D, G)$ respectively. Regarded as functions on $\widetilde{X}$, the $f_{i}$ and the $h_{j}$ vanish along the zero section $\widetilde{X} \backslash \widehat{X}$, because they are of positive degree.

According to Proposition 2.8, we can choose an equivariant open embedding $\widehat{U} \subset Z$ into an affine $\left(G \times \mathbb{K}^{*}\right)$-variety $Z$ with the following two properties: Firstly, we have $\mathcal{O}(Z) \subset \mathcal{O}(\widetilde{X})$. Secondly, the functions $f_{i}, h_{j} \in \mathcal{O}(\widehat{U})$ extend regularly to $Z$ and satisfy $\widehat{U}_{f_{i}}=Z_{f_{i}}$ and $\widehat{U}_{h_{j}}=Z_{h_{j}}$.

Now consider the induced $\mathbb{K}^{*}$-actions on the quotient spaces $Z / / T$ and $Z / / G$. As before, let $B_{T}^{0}$, $B_{G}^{0}$ be the fixed point sets of these $\mathbb{K}^{*}$-actions, and let $B_{T}^{-}, B_{G}^{-}$be the sets of nonfixed points admitting a limit for $t \rightarrow \infty$. Then, setting $A:=Z \backslash \widehat{U}$, we claim that for the respective sets of semistable points one has:

$$
\begin{aligned}
& \widehat{X} \cap q^{-1}\left(X^{\mathrm{ss}}(D, T)\right)=Z \backslash p_{T}^{-1}\left(p_{T}(A) \cup B_{T}^{0} \cup B_{T}^{-}\right), \\
& \widehat{X} \cap q^{-1}\left(X^{\mathrm{ss}}(D, G)\right)=Z \backslash p_{G}^{-1}\left(p_{G}(A) \cup B_{G}^{0} \cup B_{G}^{-}\right) .
\end{aligned}
$$

Indeed, the inclusion ' $C$ ' of the first equation is due to the facts that the intersection $\widehat{X} \cap q^{-1}\left(X \backslash Z\left(f_{i}\right)\right)$ equals $Z_{f_{i}}$, and that each $f_{i}$ by $T$-invariance and homogeneity of positive degree vanishes along the set $p_{T}^{-1}\left(p_{T}(A) \cup B_{T}^{0} \cup B_{T}^{-}\right)$. Analogously one obtains the inclusion ' $\subset$ ' for the second equation.

To see the inclusions ' $\supset$ ', we again treat the first equation for illustration purposes. The ideal of $p_{T}(A) \cup B_{T}^{0} \cup B_{T}^{-}$in $\mathcal{O}(Z / / T)$ is generated by functions $f^{\prime}$ that are homogeneous of positive degree. Since $\mathcal{O}(Z) \subset \mathcal{O}(\tilde{X})$ holds, each $f:=p_{T}^{*}\left(f^{\prime}\right)$ is a $T$-invariant homogeneous section of positive degree in $\mathcal{A}(X)$. By Proposition 2.8, we have

$$
Z_{f}=\widehat{X}_{f}=\widehat{X} \cap q^{-1}(X \backslash Z(f)) .
$$

It follows that $X \backslash Z(f)$ is affine, and hence $f$ is as in Definition 3.2. Consequently, $Z_{f}$ lies over the set of $T$-semistable points of $X$. Since the functions $f$ generate the ideal of $p_{T}^{-1}\left(p_{T}(A) \cup B_{T}^{0} \cup B_{T}^{-}\right)$, we obtain the desired inclusion.

Now, Lemmas 4.3, 4.4, and 4.5 show that the inclusion ' $\supset$ ' of the assertion is valid. The reverse inclusion is easy: Every translate $g \cdot X^{\mathrm{ss}}(D, T)$ is the set of semistable points of $g T g^{-1}$ and hence contains $X^{\mathrm{ss}}(D, G)$.

The proof of part ii is similar. As in the proof of part i, we may assume that $\Lambda$ consists of Cartier divisors. Let $\mathcal{A}$ be the associated $\Lambda$-graded $\mathcal{O}_{X}$-algebra. Consider $\widehat{X}:=\operatorname{Spec}(\mathcal{A})$ with its actions of $S:=\operatorname{Spec}(\mathbb{K}[\Lambda])$ and $G$, and the $G$-equivariant canonical map $q: \widehat{X} \rightarrow X$. Let $U \subset X$ be the ample locus of $\Lambda$, and set $\widehat{U}:=q^{-1}(U)$.

Cover $X^{\mathrm{ss}}(\Lambda, T)$ by sets $X \backslash Z\left(f_{i}\right)$ with $T$-invariant homogeneous $f_{i} \in \mathcal{A}(X)$ as in Definition 3.4. Similarly, cover $X^{\mathrm{ss}}(\Lambda, G)$ by $X \backslash Z\left(h_{j}\right)$ with $G$-invariant homogeneous $h_{j} \in \mathcal{A}(X)$. Lemma 2.8 provides an equivariant open embedding $\widehat{U} \subset Z$ into an affine $(G \times S)$-variety $Z$ with $\mathcal{O}(Z) \subset \mathcal{O}(\widehat{X})$ such that all $f_{i}, h_{j}$ extend regularly to $Z$, and satisfy $\widehat{U}_{f_{i}}=Z_{f_{i}}$ and $\widehat{U}_{h_{j}}=Z_{h_{j}}$.

For $H=T, G$, consider the quotient $p_{H}: Z \rightarrow Z / / H$ and the induced action of $S$ on $Z / / H$. We describe $X^{\mathrm{ss}}(\Lambda, H)$ in terms of these data. Let $A:=Z \backslash \widehat{U}$, and let $B_{H}^{0} \subset Z / / H$ be the set of all $y \in Z / / H$ with an infinite isotropy group $S_{y}$. We claim

$$
q^{-1}\left(X^{\mathrm{ss}}(\Lambda, H)\right)=Z \backslash p_{H}^{-1}\left(p_{H}(A) \cup B_{H}^{0}\right) .
$$

The inclusion ' $\subset$ ' follows from [Hau01, Proposition 2.3 (i)]. For the reverse inclusion, we use [Hau01, Lemma 2.4]: it tells us that the ideal of $p_{H}(A) \cup B_{H}^{0}$ in $\mathcal{O}(Z / / H)$ is generated by 


\section{GIT BASED ON WEIL DIVISORS}

$S$-homogeneous elements $f^{\prime}$ such that $\mathcal{O}(Z / / H)_{f^{\prime}}$ admits homogeneous invertible elements for almost every character of the torus $S$.

For such $f^{\prime}$, the pullback $f:=p_{H}^{*}\left(f^{\prime}\right)$ is an $H$-invariant element of $\mathcal{O}(Z)$ and hence of $\mathcal{A}(X)$, and, by Lemma 2.8, part iii, we have $\widehat{X}_{f}=Z_{f}$. Thus $q\left(\widehat{X}_{f}\right)=X \backslash Z(f)$ is affine, and we see that $f$ is as in Definition 3.4. Hence, $q^{-1}\left(X^{\mathrm{ss}}(\Lambda, H)\right) \supset Z_{f}$ holds, which finally gives the claim.

Now, $B_{H}^{0} \subset Z / / H$ is the union of the fixed point sets $B_{H}^{0}(\mu)$ of all one-parameter subgroups $\mu: \mathbb{K}^{*} \rightarrow S$. Lemmas 4.3 and 4.4 tell us that

$$
p_{G}^{-1}\left(p_{G}(A)\right)=\bigcup_{g \in G} g \cdot p_{T}^{-1}\left(p_{T}(A)\right), \quad p_{G}^{-1}\left(B_{G}^{\circ}(\mu)\right)=\bigcup_{g \in G} g \cdot p_{T}^{-1}\left(B_{T}^{\circ}(\mu)\right) .
$$

Together with (2), this gives ' $\supset$ ' in the assertion. The reverse inclusion is due to the fact that $g \cdot X^{\mathrm{ss}}(\Lambda, T)$ is the set of semistable points of $g T g^{-1} \subset G$.

\section{Actions of semisimple groups}

In this section, we apply our results to actions of semisimple groups. This gives generalizations of several results presented in [BBS92], [BBS95] and [Hau03]. We work with the following notions of maximality (compare [BBS92] and [Hau03]).

Definition 5.1. Let $G$ be a reductive group, let $X$ be a $G$-variety, and let $U \subset X$ be a $G$-invariant open subset. We say that

i) $U$ is a $q p$-maximal $G$-set if there is a good quotient $U \rightarrow U / / G$ with $U / / G$ quasiprojective, and $U$ is not a $G$-saturated subset of a properly larger $U^{\prime} \subset X$ admitting a good quotient $U^{\prime} \rightarrow U^{\prime} / / G$ with $U^{\prime} / / G$ quasiprojective,

ii) $U$ is a $d$-maximal $G$-set if there is a good quotient $U \rightarrow U / / G$ with $U / / G$ divisorial, and $U$ is not a $G$-saturated subset of a properly larger $U^{\prime} \subset X$ admitting a good quotient $U^{\prime} \rightarrow U^{\prime} / / G$ with $U^{\prime} / / G$ divisorial.

In the sequel, $G$ is a connected semisimple group, $T \subset G$ a maximal torus, and $N \subset G$ the normalizer of $T$ in $G$. Moreover, $X$ is a normal $G$-variety. The first result is a further HilbertMumford type statement. It generalizes [BBS95, Corollary 1], and the results in the case $G=\mathrm{SL}_{2}$ given in [BBS92, Theorem 9] and [Hau03, Theorem 2.2].

Theorem 5.2. Let $U \subset X$ be an $N$-invariant open subset of $X$, and let $W(U)$ denote the intersection of all translates $g \cdot U$, where $g \in G$.

i) If $U \subset X$ is a qp-maximal $T$-set, then $W(U)$ is open and $T$-saturated in $U$, and there is a good quotient $W(U) \rightarrow W(U) / / G$ with $W(U) / / G$ quasiprojective.

ii) If $U \subset X$ is a d-maximal $N$-set, then $W(U)$ is open and $T$-saturated in $U$, and there is a good quotient $W(U) \rightarrow W(U) / / G$ with $W(U) / / G$ divisorial.

The proof of this theorem consists of combining the Hilbert-Mumford Theorem (Theorem 4.1) with the following observation.

Proposition 5.3. Let $U \subset X$ be an $N$-invariant open subset.

i) If $U$ is a qp-maximal $N$-set, then there exists a $G$-linearized Weil divisor $D$ on $X$ with $U=$ $X^{\mathrm{ss}}(D, N)$.

ii) If $U$ is a d-maximal $N$-set, then there is a $G$-linearized group $\Lambda \subset \operatorname{WDiv}(X)$ with $U=$ $X^{\mathrm{ss}}(\Lambda, N)$. 


\section{J. Hausen}

Proof. For illustrative purposes we prove the first assertion. By Theorem 3.3, part ii, there is a canonically $N$-linearized Weil divisor $D$ on $X$ such that $U$ is $N$-saturated in $X^{\mathrm{ss}}(D, N)$. By qpmaximality of $U$, this implies $U=X^{\mathrm{ss}}(D, N)$. We show now that, after possibly replacing $D$ with a positive multiple, the $N$-linearization extends to a $G$-linearization.

Let $Z$ be a $G$-equivariant completion of $X$ (see [Sum74, Theorem 3]). Applying equivariant normalization, we achieve that $Z$ is normal. By closing the support, we extend $D$ to a Weil divisor $E$ of $Z$. Then $E$ is $N$-invariant and hence, by Proposition 1.7, it is canonically $N$-linearized.

Proposition 1.6 tells us that after replacing $E$ (and $D$ ) with a suitable multiple, we can choose a $G$-linearization of $E$. Since we have $\mathcal{O}(Z)=\mathbb{K}$ and the character group of $N$ is finite, Proposition 1.8, part ii says that, after possibly passing to a further multiple, the $G$-linearization of $E$ induces the canonical $N$-linearization of $E$ over $Z$. Restricting to $X \subset Z$, we obtain the assertion.

Note that this proposition is the place where semisimplicity of $G$ came in. In the proof, we made essential use of the fact that the character group of $N$ is finite.

Proof of Theorem 5.2. Note first that in the setting of part i, the induced action of the Weyl group $N / T$ on $U / / T$ admits a geometric quotient with a quasiprojective quotient space. The composition of the quotients by $T$ and $N / T$ is a good quotient $U \rightarrow U / / N$. It follows that $U$ is a qp-maximal $N$-set.

Now, for part i, choose a $G$-linearized semigroup $\Lambda=\mathbb{N} D$, and, for part ii, a $G$-linearized group $\Lambda \subset \mathrm{WDiv}(X)$ as provided by Proposition 5.3. By the definition of semistability, we have

$$
X^{\mathrm{ss}}(\Lambda, G) \subset \bigcap_{g \in G} g \cdot X^{\mathrm{ss}}(\Lambda, N) \subset \bigcap_{g \in G} g \cdot X^{\mathrm{ss}}(\Lambda, T) .
$$

From Theorem 4.1 we infer that also the reverse inclusions hold. This gives the assertion.

In the case of complete quotient spaces, the approach via Weil divisors finally turns out to be a detour: here everything can be done in terms of line bundles. More precisely, we have the following generalization of [BBS95, Theorem 1]; compare also [BBS95, Remark, p. 965].

Theorem 5.4. Let $U \subset X$ be an $N$-invariant open subset admitting a good quotient $U \rightarrow U / / T$ with $U / / T$ projective. Then there is an ample $G$-linearized line bundle $L$ on $X$ such that $U=X^{\mathrm{ss}}(L, T)$ holds. Moreover, we have $X=G \cdot U$, and $X$ is a projective variety.

Combining this result with [MFK94, Theorem 2.1] gives the following supplement to the HilbertMumford Theorem (Theorem 5.2).

Corollary 5.5. Let $U \subset X$ be as in Theorem 5.4. Then the intersection $W(U)$ of all translates $g \cdot U, g \in G$, is an open $T$-saturated subset of $U$, there is a good quotient $W(U) \rightarrow W(U) / / G$, and $W(U) / / G$ is projective.

We come to the proof of Theorem 5.4. A first ingredient is an observation due to Białynicki-Birula and Święcicka concerning semisimple group actions on the projective space.

Lemma 5.6. Let $G$ act on $\mathbb{P}_{n}$. Then the translates $g \cdot \mathbb{P}_{n}^{\text {ss }}(\mathcal{O}(1), T)$, where $g \in G$, cover $\mathbb{P}_{n}$.

Proof. Consider the complement $Y$ of the union of all translates $g \cdot \mathbb{P}_{n}^{\text {ss }}(\mathcal{O}(1), T)$, where $g \in G$. Then $Y$ is empty, because otherwise [BBS95, Lemma, p. 963] would provide a $T$-semistable point in some irreducible component of $Y$.

The second ingredient of the proof is the following refinement of Sumihiro's Embedding Theorem (compare [Sum74, Theorem 1] and [MFK94, Proposition 1.7]). 


\section{GIT BASED ON WEIL DIVISORS}

Lemma 5.7. Let $D$ be a $G$-linearized Cartier divisor. If $X=G \cdot X^{\mathrm{ss}}(D, T)$ holds, then there is a $G$-equivariant locally closed embedding $X \subset \mathbb{P}_{n}$ such that $X^{\mathrm{ss}}(D, T)$ is $T$-saturated in $\bar{X} \cap \mathbb{P}_{n}^{\text {ss }}(\mathcal{O}(1), T)$, where $\bar{X}$ is the closure of $X$ in $\mathbb{P}_{n}$.

Proof. Let $\mathcal{A}$ be the graded $\mathcal{O}_{X}$-algebra associated to $D$, and let $U:=X^{\mathrm{ss}}(D, T)$. Since we assumed $X=G \cdot U$, Proposition 2.5 tells us that the divisor $D$ is in fact ample. Moreover, replacing $D$ with a multiple, we may even assume that $D$ is very ample, and that there are $T$-invariant $f_{1}, \ldots, f_{r}$ $\in \mathcal{A}_{D}(X)$ such that the sets $X \backslash Z\left(f_{i}\right)$ are affine and cover $U$.

Choose any $G$-invariant vector subspace $M \subset \mathcal{A}_{D}(X)$ of finite dimension such that $f_{1}, \ldots, f_{r}$ $\in M$ holds, and the corresponding morphism $\imath: X \rightarrow \mathbb{P}(N)$ is a locally closed embedding, where $N$ is the dual $G$-module of $M$. Then $\imath$ is $G$-equivariant, and $\mathcal{A}_{D}$ equals as a $G$-sheaf the pullback of $\mathcal{O}(1)$. Moreover, by construction, the $f_{i}$ extend to $T$-invariant sections of $\mathcal{O}(1)$.

Proof of Theorem 5.4. First note that $U$ is as well a qp-maximal $N$-set. Thus we can choose a $G$-linearized Weil divisor $D$ on $X$ as in Proposition 5.3, part i. By Proposition 2.5, $D$ is an ample Cartier divisor on $X_{0}:=G \cdot U$. In particular, on $X_{0}$ the $G$-sheaf $\mathcal{A}_{D}$ is the sheaf of sections of a $G$-linearized line bundle.

Now choose a locally closed $G$-equivariant embedding $X_{0} \subset \mathbb{P}_{n}$ as in Lemma 5.7 , and let $\bar{X}_{0}$ denote the closure of $X_{0}$ in $\mathbb{P}_{n}$. Since $U / / T$ is complete, we obtain

$$
U=\bar{X}_{0} \cap \mathbb{P}_{n}^{\mathrm{ss}}(\mathcal{O}(1), T) .
$$

Moreover, from Lemma 5.6 we infer that the translates $g \cdot U$, where $g \in G$, cover $\bar{X}_{0}$. But this means that we have $X_{0}=\bar{X}_{0}$. In particular $X_{0}$ is projective, $X=X_{0}$ holds, and $D$ is ample.

\section{ACKNowledgement}

I would like to thank the referee for helpful suggestions and comments on the earlier versions of this paper.

\section{REFERENCES}

ACH01 A. A'Campo-Neuen and J. Hausen, Toric prevarieties and subtorus actions, Geom. Dedicata $\mathbf{8 7}$ (2001), 35-64.

ACH02 A. A'Campo-Neuen and J. Hausen, Quotients of divisorial toric varieties, Michigan Math. J. 50 (2002), 101-123.

BGI71 P. Berthelot, A. Grothendieck and L. Illusie et al., Théorie des intersections et théorème de Riemann-Roch, in Séminaire de géométrie algébrique du Bois-Marie 1966-67 (SGA 6), Lecture Notes in Mathematics, vol. 225 (Springer, Berlin, 1971).

BB02 A. Białynicki-Birula, Algebraic quotients, in Encyclopedia of Mathematical Sciences, eds R. V. Gamkrelidze and V. L. Popov, vol. 131 (Springer, Berlin, 2002), 1-82.

BBS92 A. Białynicki-Birula and J. Święcicka, On complete orbit spaces of SL(2)-actions, II, Colloq. Math. 63 (1992), 9-20.

BBS95 A. Białynicki-Birula and J. Święcicka, On projectivity of good quotients, J. Algebra 177 (1995), 961-966.

Bor63 M. Borelli, Divisorial varieties, Pacific J. Math. 13 (1963), 375-388.

Bir71 D. Birkes, Orbits of linear algebraic groups, Ann. of Math. (2) 93 (1971), 459-475.

FI73 R. Fossum and B. Iversen, On Picard groups of algebraic fibre spaces, J. Pure Appl. Algebra 3 (1973), 269-280.

Ful93 W. Fulton, Introduction to toric varieties, The 1989 William H. Roever lectures in geometry, Annals of Mathematics Studies, vol. 131 (Princeton University Press, Princeton, NJ, 1993). 


\section{GIT BASED ON WEIL DIVISORS}

GD61 A. Grothendieck and J. Dieudonné, Éléments de géométrie algébrique, II, Étude globale élémentaire de quelques classes de morphismes, Publ. Math. Inst. Hautes Études Sci. 8 (1961).

Ham00 H. A. Hamm, Very good quotients of toric varieties, in Real and complex singularities. Proc. 5th workshop, Sao Carlos, Brazil, 27-31 July 1998, eds J. W. Bruce et al., Chapman Hall/CRC Research Notes in Mathematics, vol. 412 (Chapman \& Hall/CRC, Boca Raton, FL, 2000), 61-75.

Hau01 J. Hausen, A generalization of Mumford's geometric invariant theory, Documenta Math. 6 (2001), $571-592$.

Hau03 J. Hausen, A Hilbert-Mumford criterion for $\mathrm{SL}_{2}$-actions, Colloq. Math. 97 (2003), 151-161.

Hum81 J. E. Humphreys, Linear algebraic groups, Graduate Texts in Mathematics, vol. 21, Corrected second printing (Springer, Berlin, 1981).

KKLV89 F. Knop, H. Kraft, D. Luna and T. Vust, Local properties of algebraic group actions, in Algebraische Transformationsgruppen und Invariantentheorie, DMV Seminar, Band 13 (Birkhäuser, Basel 1989), $63-75$.

MFK94 D. Mumford, J. Fogarty and F. Kirwan, Geometric invariant theory, third edition, Ergeb. Math. Grenzgeb. (2), vol. 34 (Springer, Berlin, 1994).

Sch03 A. Schmitt, A simple proof for the finiteness of GIT-quotients, Proc. Amer. Math. Soc. 131 (2003), $359-362$.

Ses72 C. S. Seshadri, Quotient spaces modulo reductive algebraic groups, Ann. of Math. (2) 95 (1972), $511-556$.

Sum74 H. Sumihiro, Equivariant completion, J. Math. Kyoto Univ. 14 (1974), 1-28.

Sum75 H. Sumihiro, Equivariant completion, II, J. Math. Kyoto Univ. 15 (1975), 573-605.

Wlo99 J. Włodarczyk, Maximal quasiprojective subsets and the Kleiman-Chevalley Quasiprojectivity Criterion. J. Math. Sci. Univ. Tokyo 6 (1999), 41-47.

Jürgen Hausen hausen@mfo.de

Mathematisches Forschungsinstitut Oberwolfach, Schwarzwaldstraße 9-11, 77709 OberwolfachWalke, Germany 